\title{
The prevalence and risk factors of work- related musculoskeletal disorders among adults in Ethiopia: a study protocol for extending a systematic review with meta- analysis of observational studies
}

\author{
Tsiwaye Gebreyesus ${ }^{1}$, Kalkidan Nigussie ${ }^{2}$, Moges Gashaw ${ }^{2}$ and Balamurugan Janakiraman ${ }^{2^{*}}$ (iD
}

\begin{abstract}
Background: Work-related musculoskeletal disorders impose a significant and most often underappreciated burden to the individual, nation, healthcare system, and society as a whole. To the best of our knowledge, there is a lack of reliable estimates on the prevalence of work-related musculoskeletal disorders in Ethiopia. The objective of this study will be to assess the existing literature on the prevalence rates and determinant factors of work-related musculoskeletal disorders in Ethiopia.

Method: We will search PubMed/MEDLINE, Embase, SCOPUS, PsycINFO, PEDro, and Ebsco (from January 2000 onwards). Gray literature will be identified through searching Google Scholar and dissertation databases. Observational studies reporting on the prevalence of work-related musculoskeletal disorders among adult Ethiopians will be included. The primary outcome will be the prevalence of work-related musculoskeletal disorders. Secondary outcomes will be the prevalence of any risk factors in association with work-related musculoskeletal disorders. Two reviewers will independently screen all citations, full-text articles, and abstract data. The study methodological quality (or bias) will be appraised using an appropriate tool. If feasible, we will conduct random effects meta-analysis of observational data. Subgroup analyses will be conducted to explore the potential sources of heterogeneity (e.g., gender, sample size, type of occupation). Publication bias and heterogeneity between the included studies will also be assessed and reported.

Discussion: This systematic review will provide a synthesis of the literature on the prevalence of work-related musculoskeletal disorders and their risk factors in Ethiopia. The results of this review could help the policymakers in occupational health and healthcare sectors in identifying priority areas for interventions in work-related musculoskeletal disorders and will also serve as a baseline for the decision-making processes of musculoskeletal health promotion, work exposure implementations, and prevention programs in workplaces.

(Continued on next page)
\end{abstract}

\footnotetext{
* Correspondence: bala77physio@gmail.com

${ }^{2}$ Department of Physiotherapy, School of Medicine, College of Medicine and Health Sciences, and Specialized Hospital, University of Gondar, P.O. Box No. 196, Gondar, Ethiopia

Full list of author information is available at the end of the article
}

C C The Author(s). 2020 Open Access This article is licensed under a Creative Commons Attribution 4.0 International License, which permits use, sharing, adaptation, distribution and reproduction in any medium or format, as long as you give appropriate credit to the original author(s) and the source, provide a link to the Creative Commons licence, and indicate if changes were made. The images or other third party material in this article are included in the article's Creative Commons licence, unless indicated otherwise in a credit line to the material. If material is not included in the article's Creative Commons licence and your intended use is not permitted by statutory regulation or exceeds the permitted use, you will need to obtain permission directly from the copyright holder. To view a copy of this licence, visit http://creativecommons.org/licenses/by/4.0/ The Creative Commons Public Domain Dedication waiver (http://creativecommons.org/publicdomain/zero/1.0/) applies to the data made available in this article, unless otherwise stated in a credit line to the data. 
(Continued from previous page)

Systematic review registration: PROSPERO, CRD42020164240

Keywords: Prevalence, Work-related musculoskeletal pain, Population-based observational studies, Systematic review protocol, Ethiopia

\section{Background}

Musculoskeletal disorders (MSDs) are common workplace health problems characterized by a range of symptoms like pain, ache, and discomfort in bodily regions [1, 2]. Musculoskeletal disorders (MSDs) are impairments of body structures such as muscles, tendons, fascia, ligaments, joints, nerves, bones, or a localized blood circulation system either caused or aggravated by poor fitness, and poor health habits, but a major proportion of MSDs are caused by physical work exposures [3, 4]. And these impairment occurs secondary to sudden injuries and cumulative trauma, with the latter being the most common mechanism behind work-related musculoskeletal disorders (WRMSDs) [1, 3, 5].

According to Global Burden of Diseases (GBD), injuries, and risk factors study 2017, between 2007 and 2017, among the three causes which are observed to have resulted in a further increase in the number of all-age years lived with disability (YLDs), low back pain (LBP) is attributable to a further increase of $17,5 \%$ (95\% UI 16.2-19.0), While the YLDs counts are heavily concentrated in working-age (i.e., from 20-54 years), a pattern which is particularly evident among causes such as musculoskeletal disorders, mental disorders, and neurological disorders which sum to more than $45 \%$ of all YLDs in these age groups $[6,7]$. This finding is notable as the working-age group in countries with low sociodemographic index (SDI) has a considerable number of years to live, and musculoskeletal disorders of even lower to moderate disability weights can cause substantial non-fatal burden and lost human capital [8]. Moreover, musculoskeletal disorders being three of the top 10 conditions in terms of disability and burden from noncommunicable diseases (NCDs) in developing countries. Total disability-adjusted life years (DALYs) from low back and neck pain also increased, rising by more than $17 \%$ from 2000 to 2017 [9-11].

GBD 2017 reported that MSD's are the second most common cause of years lost to disability, while years of life lost are declining in sub-Saharan. Further, the burden of MSD will only continue to increase as the sub-Saharan population ages [7, 12]. Work-related musculoskeletal disorders (WRMSDs) and other musculoskeletal conditions remain less prioritized and empirically unrepresented in low-middle income countries (LMICs), particularly in Ethiopia due to focus on more pressing and lifethreatening health issues like NCDs and infectious diseases [13]. A previous systematic review has however reported on the prevalence of WRMSDs in Africa which included a majority of studies from South Africa and Nigeria. The prevalence of WRMSDs varies from 13 to 92\% in South Africa and Ghana respectively [14]. Empirically, racial, economic, and social homogeneity is not a feature of Africa and may underlie the reported difference in the prevalence. Hence, it is logical, therefore, to argue that genetic diversity, differences in social structure, economics, and other Ethiopian specific factors may influence the WRMSDs prevalence among Ethiopians. Further, musculoskeletal disorders remain a major global health concern and an immense burden for LMICs like Ethiopia where health budgets are already constrained and channeled towards the life-threatening conditions. Studies have shown that musculoskeletal pain prevalence among the working population in Ethiopia varies from $35 \%$ to $74.5 \%$ [13-22].

Though in the past decade many individual studies have reported the prevalence of regional pain, general WRMSDs, and factors associated with the working Ethiopian population, to our knowledge, no published systematic review reporting on the prevalence of workrelated musculoskeletal on Ethiopian adult population exists. The consensus of data from this review will help occupational health-related policymakers, healthcare professionals and program managers in developing countries in particular Ethiopia, to gain a better understanding of the prevalence, causes, and trends to build a better evidence-based occupational musculoskeletal health and disorders prevention programs. The objective of this study will be to assess the prevalence of workrelated musculoskeletal disorders and their risk factors among the working adult population in Ethiopia.

Specific review questions are the following:

- What is the prevalence of musculoskeletal disorders in the categories of bodily regions, for different occupational settings and subgroups in Ethiopia according to internationally published studies?

- What are the occupational factors and patterns (pain sites, frequency, distribution across body) of musculoskeletal pain, what are the factors that cause and/or related to good or poor musculoskeletal health for different occupational settings and subgroups in the adult working population in Ethiopia according to internationally published studies? 
- What is the methodological quality of the prevalence studies, intending to identify approaches to improve future research quality?

\section{Methods}

\section{Systematic review registration and reporting}

The present review protocol has been registered within the PROSPERO database (registration number CRD42020164240) and is being reported in accordance with the reporting guidance provided in the Preferred Reporting Items for Systematic Reviews and MetaAnalyses Protocols (PRISMA-P) statement [23, 24] (see checklist in Additional file 1) and Meta-analysis Of Observational Studies in Epidemiology (MOOSE) reporting guideline [25].

\section{Data sources and search strategies}

The primary source of literature will be a structured search of electronic databases (from January 2000 onwards, as we are primarily interested in the contemporary literature): PubMed/MEDLINE, Embase, SCOPUS, PsycINFO, PEDro, and Ebsco. The secondary source of potentially relevant material will be a search of the gray or difficult to locate literature, including Google Scholar, dissertation databases and other relevant databases (e.g., World Health Organization, Centers for Disease Control and Prevention, Work Place Health Promotion). We will perform hand searching of the reference lists of included studies, relevant reviews or other relevant documents. The search will include a broad range of terms and keywords related to musculoskeletal disorders, workplace and occupation, prevalence and the geographical area 'Ethiopia'. A draft search strategy for PubMed/MEDLINE is provided in Additional file 2. Only studies published in English (or with an English version of the document) will be eligible. Moreover, the corresponding authors will be contacted by mail whenever a need arise or for any difficulties faced during data extraction.

\section{Eligibility criteria}

Studies will be selected based on the following criteria: participants, condition or outcome(s) of interest, study design, and context.

Participants (population): We will include studies involving adult population ( $\geq 18$ years of age, regardless of sex) working in governmental sectors or private sectors or self-employed in Ethiopia.

\section{Condition or outcome(s) of interest}

The primary outcome will be the prevalence (e.g., point prevalence, period prevalence, prevalence rate) of WRMSDs (including neck disorders, low back disorders, or other chronic disorders) indicating the number of people that have the disorder divided by the population number at a given point in time, presented often as a (prevalence) proportion. We will use the authors' reported definitions using standardized scales or questionnaires (e.g., standardized nordic questionnaire, Dutch musculoskeletal questionnaire, or visual analog scale) providing sufficient information to calculate the prevalence. Secondary outcomes will be to identify the risk factors of musculoskeletal disorders (e.g., psychosocial factors, posture, heavy physical work, workplace-related factors, and smoking). When outcome results (e.g., proportion and $95 \% \mathrm{CI}$ ) are not directly reported and it is feasible, we will calculate estimates from the number of cases and sample size mentioned in each single study.

\section{Study design and context}

Eligible studies will be observational studies (cohort, cross sectional, or health surveys) reporting prevalence data using validated or non-validated tools and conducted in the Ethiopian working population. Crosssectional studies will be the most appropriate study design to determine the pooled prevalence of the workrelated musculoskeletal disorders. For cohort studies, only the first phase (cross-sectional) data will be considered. We will exclude studies published in non-English, reviews, commentaries, conference abstracts, letter to editors, non-human articles, and studies conducted outside of Ethiopia.

\section{Study screening and selection for inclusion in the review}

The titles and abstracts of articles retrieved from the search of different databases will be stored and managed in a Zotero version 5 reference manager and we will remove the duplicate records from it. Two reviewers (B) and $\mathrm{KN}$ ) will independently review the titles and abstract part of all the articles, the full text of the potentially eligible article will be assessed for compliance and analyzed for inclusion. Disagreements or conflicts throughout the review process will be resolved by consensus and if needed, by requesting the opinion of the third and fourth reviewers. The consistency of the selection process and quality assessment across the reviewers will be ensured by calculating the level of inter-rater agreement (Kappa statistics) [26]. A PRISMA flowchart showing details of the studies included and excluded at each stage of the study selection process will be provided.

\section{Data extraction and management}

Once eligible studies are identified, two independent reviewers will extract the data using a prepared standardized data extraction form. Reviewers will not perform data extraction from an article of which they were (co-) authors to eliminate possible bias. Data such as first author's last name, year of publication, study location within Ethiopia, sample size, response rate, the reason 
for non-response, number of events, regions of pain reported, data on prevalence, recall period, ascertainment of outcome measures, risk factor or protective factors determined by each study along with their respective odds ratio (OR) and 95\% confidence interval, and information needed for the risk of bias assessment will be extracted (Table 1). The findings of the review will be illustrated through figures and tables.

\section{Risk of bias and quality assessment}

Two review authors (BJ and KN) will independently assess the quality of all included studies using the Newcastle-Ottawa Quality Assessment tool adapted for cross-sectional studies [27, 28]. The tool will be further adapted for use in this review (Additional file 3). Discrepancies of aggregate or total scores will be resolved by the third reviewer (TG) after a detailed evaluation of the source of the discrepancy. The tool contains three domains; selection of participants (3 items), quality of data (4 items), and definition of work-related musculoskeletal disorders (3 items). For the purpose of this review, all the items in the appraisal tool will be equally weighted and so the total score will be 10 . There will be no subminimum score criteria for inclusion of studies.

\section{Data synthesis}

The prevalence rate, the logarithm of prevalence, and standard error (SE) of the logarithm of prevalence will be computed. Correspondingly, for the factors associated, the logarithm of OR and SE of the logarithms of OR will be calculated. The pooled prevalence (proportion) of musculoskeletal disorders or musculoskeletal pain-related work and the pooled odds ratios (OR) of

Table 1 Data items that will be extracted

1. Publication details: title, journal, author, year, city/region in Ethiopia, where the study was conducted, type of publication, and source of funding, if any

2. Design: type of study design (observational studies, cross sectional, cohort, case control, others); aims or objective of the study, method of data collection, outcome variable (measures and operational definition), response rate, recruitment and sampling method, eligibility (inclusion and exclusion criteria)

3. Study participants information's: sample size, population characteristics including settings, role in the institution, age, gender, ethnicity, education level, demographic information's, behavioral information's, physical measurements, comorbidities, other healthrelated characteristics, employment details, work and workenvironment related characteristics, bodily distribution of pain

4. Data for outcome variables: all reported estimates, or sufficient data to calculate an estimate of the point prevalence, cumulative incidence, and incidence rate of musculoskeletal disorders in categories of bodily distribution like low back pain, neck pain, shoulder pain, elbow pain, wrist and hand pain, hip pain, knee pain, ankle pain, etc

5. Limitations: bias (selection, response, information), limitation of outcome measures/tools, and limitation reported by authors associated factors with a $95 \%$ confidence interval will be calculated using random-effects and quality effects model. The quality-effects meta-analysis [29] will be used to examine how the quality of each study influenced the pooled estimate compared with the results from the random effects [30]. The quality scores of each included study will be incorporated in the calculation of study weight to improve the robustness and help minimize the estimator variance and subjectivity in quality assessment. The presence of heterogeneity among studies will be quantified by estimating variance using both Cochrane's Q statistics and the $I^{2}$ statistics. $I^{2}$ statistics is the proportion of the variation in the estimates of prevalence due to genuine variation in prevalence rather than sampling error [31, 32]. Funnel plots will be used to assess small study effects or publication bias [33] by inspection of asymmetry and in addition Egger's regression test $(p<0.05)$ [34] and Beggs equations will be computed to declare publication bias. Double arsine transformation will be used in the case of variance instability [35].

Possible subgroups will be identified based on the study characteristics and population characteristics. Subgroup analysis will be performed to determine the source of heterogeneity attributed to gender, sample size, place of study, study setting, study design, year of publication, outcome tool used, type of occupation, and region of pain. Sensitivity analysis will be performed after excluding each study one by one and the pooled estimate will be calculated for the remaining studies. All statistical analyses will be performed using Meta XL version 5.3 [36] and STATA 15 Metaprop package [37]. Quantitative data from different designs, variability in prevalence (e.g., cohort, case-control studies) will not be combined, nor unadjusted and adjusted models. Transformation of rates to their natural logarithm, with corresponding standard errors (SEs) will be entered into meta-analyses. When quantitative synthesis is not feasible, we will retain the studies for narrative synthesis.

\section{Timeline, presenting, and reporting of the results}

The review process will commence only after the final peer-review comments are received, and the protocol accepted for publication. The study selection step by step process will be outlined in a flow diagram and the reasons for exclusion will also be mentioned. The study characteristics, risk of bias, and quality assessment of the included studies will be presented in tables. Forest plots will be used to display the pooled estimates of prevalence proportions.

\section{Discussion}

In the past decade, institutional and population-based studies on work-related musculoskeletal disorders have 
grown in Ethiopia. This review will aim to provide a current overview of the prevalence of musculoskeletal disorders in working-aged Ethiopian adults from various occupations, cultures, and age groups to provide a better understanding of musculoskeletal health and its effect on their life. This review will also synthesize all possible available information for occupational health policymakers, healthcare workers, and identify priority areas for interventions in work-related musculoskeletal disorders in Ethiopia.

Furthermore, this will be the first systematic review and meta-analysis that will determine the pooled prevalence of musculoskeletal disorders among the adult working population in Ethiopia. In addition, this paper will also identify factors associated with work-related musculoskeletal disorders among Ethiopians. Though WRMSD is not life-threatening, the initiative to include WRMSDs is critical in developing countries rather than being stand-alone. We believe that the inclusion of WRMSDs in Ethiopia will avoid efforts doubling and wasting of resources in the future. The rationale for the need to conduct this review is the previous two Africanbased reviews reporting on WRMSDs (2015) and low back pain (2007) were limited by not including any studies from Ethiopia [14, 38] and in addition, Ethiopianspecific factors like social structure, genetic diversity, nutritional status, and work culture will surely add more insight on the Ethiopian working population.

Among the potential challenges we anticipate are most importantly at review level decisions as to studies reporting the prevalence of headache should be classified as dealing with musculoskeletal disorders or not, as well as at study level by heterogeneity in occupations, measurement tools, and region of pain. We will strive to mitigate these anticipated and future challenges by highlighting decision criteria, limitations, gaps in knowledge, and caution in the interpretation of findings.

\section{Strengths and limitations}

The strengths of this review include established national-level consensus of data, as well as a systematic and transparent approach. Our search will be conducted in close collaboration with the specialized research librarian, the screening and extraction will be performed by two researchers using a standardized extraction form. The inter-rater agreement between reviewers in the selection process will be statistically assessed. Furthermore, this review will also be the first one to consensus data on work-related musculoskeletal disorders in Ethiopia.

We anticipate that publication bias and heterogeneity may pose a limitation for this review. There are also possibilities of reporting and response set bias. In addition, this study is restricted to published reports in the English language.

\section{Dissemination}

The findings of this review will be disseminated through publication in peer-reviewed journals and conference presentations at the relevant venue.

\section{Supplementary information}

Supplementary information accompanies this paper at https://doi.org/10. 1186/s13643-020-01403-9.

Additional file 1. PRISMA-P (Preferred Reporting Items for Systematic review and Meta-Analysis Protocols) 2015 checklist: recommended items to address in a systematic review protocol.

Additional file 2. Example search that will be used for screening of articles in PubMed database.

Additional file 3. Adapted table of Newcastle-Ottawa quality assess ment tool.

\section{Abbreviations}

BoD: Burden of diseases; DALYs: Disability-adjusted life years; GBD: Global burden of disease; LBP: Low back pain; LMICs: Low-middle income countries; MeSH: Medical Subject Heading; MOOSE: Meta-analysis Of Observational Studies in Epidemiology; MSDs: Musculoskeletal disorders; NCDs: Noncommunicable diseases; OR: Odds ratio; PRISMA-P: Preferred Reporting Items for Systematic Reviews and Meta-analysis Protocols; SDI: Socio-demographic index; SE: Standard error; WHO: World Health Organization; WRMSDs: Workrelated musculoskeletal disorders; YLD: Years lived with disability

\section{Acknowledgements}

We thank Mekelle University, School of Medicine, and University of Gondar, School of Medicine for their help in access to University subscribed web databases.

Authors' contributions

TG conceived the research question, edited the protocol, and designed the study, MG, KN, and BJ designed the search strategy, will participate in the search process, appraise the quality of the articles, and extract needed data independently. TG will analyze and interpret the results. All authors read and approve this protocol before sending it for the publication. BJ will be the guarantor, supervisor, and corresponding person on behalf of all the reviewers.

\section{Author's information}

TG is working as a Lecturer of Physiotherapy, School of Medicine, College of Medicine and Health Sciences, Mekelle University, Mekelle, Ethiopia. BJ Assistant professor of Physiotherapy \& Senior clinical consultant physiotherapist and MG, KN lecturer in Physiotherapy, Senior Physiotherapist, School of Medicine, College of Medicine and Health Sciences and Specialized Hospital, University of Gondar, Gondar, Ethiopia.

\section{Funding}

No funding agency or organization. This systematic review and meta-analysis will be done without the help of government/research organizations. Rather it is by the motivation of the authors.

Availability of data and materials

Not applicable

Ethical approval and consent to participate Not required

Consent for publication

All authors consent and approve the manuscript for publication.

Competing interests

The authors declare no competing interests 


\section{Author details}

'Department of Physiotherapy, School of Medicine, College of Health Sciences and Ayder Comprehensive Specialized Hospital, Mekelle University, Mek'ele, Ethiopia. 'Department of Physiotherapy, School of Medicine, College of Medicine and Health Sciences, and Specialized Hospital, University of Gondar, P.O. Box No. 196, Gondar, Ethiopia.

Received: 17 March 2020 Accepted: 29 May 2020

Published online: 08 June 2020

\section{References}

1. Al-Hourani Z, Nazzal M, Khader Y, Almhdawi K, Bibars AR. Work-related musculoskeletal disorders among Jordanian dental technicians: prevalence and associated factors. Work. 2017;56(4):617-23.

2. Bethge M. Work-Related Medical Rehabilitation. Rehabilitation (Stuttg). 2017 Feb;56(1):14-21

3. Roquelaure Y, Bodin J, Descatha A, Petit A. Musculoskeletal disorders: how to recognize them as occupational disease. Rev Prat. 2018;68(10):1132-4.

4. Gómez-Galán M, Pérez-Alonso J, Callejón-Ferre Á-J, López-Martínez J. Musculoskeletal disorders: OWAS review. Ind Health. 2017;55(4):314-37.

5. Oranye NO, Bennett J. Prevalence of work-related musculoskeletal and nonmusculoskeletal injuries in health care workers: the implications for work disability management. Ergonomics. 2018;61(3):355-66.

6. Safiri S, Kolahi A-A, Hoy D, Buchbinder R, Mansournia MA, Bettampadi D, et al. Global, regional, and national burden of neck pain in the general population, 1990-2017: systematic analysis of the Global Burden of Disease Study 2017. bmj. 2020;368

7. Vos T, Allen C, Arora M, Barber RM, Bhutta ZA, Brown A, et al. Global, regional, and national incidence, prevalence, and years lived with disability for 310 diseases and injuries, 1990-2015: a systematic analysis for the global burden of disease study 2015. Lancet. 2016;388(10053):1545-602.

8. Human capital: the greatest asset of economies on the rise [Internet]. World Bank. [cited $2020 \mathrm{Apr}$ 16]. Available from: https://www.worldbank.org/en/ news/opinion/2017/04/03/human-capital-the-greatest-asset-of-economieson-the-rise.

9. Hoy DG, Smith E, Cross M, Sanchez-Riera L, Buchbinder R, Blyth FM, et al. The global burden of musculoskeletal conditions for 2010: an overview of methods. Ann Rheum Dis. 2014;73(6):982-9.

10. Hoy DG, Smith E, Cross M, Sanchez-Riera L, Blyth FM, Buchbinder R, et al. Reflecting on the global burden of musculoskeletal conditions: lessons learnt from the global burden of disease 2010 study and the next steps forward. Ann Rheum Dis. 2015;74(1):4-7.

11. Storheim K, Zwart J-A. Musculoskeletal disorders and the global burden of disease study; 2014.

12. Fink DL, Oladele D, Etomi O, Olaosebikan H, Dey ID, Adelowo OO. We must harness technology to deliver the musculoskeletal disease epidemiology that is urgently needed across sub-Saharan Africa. Clin Rheumatol. 2018; 37(5):1439-40.

13. Woolf AD, Brooks P, Åkesson K, Mody GM. Prevention of musculoskeletal conditions in the developing world. Best Pract Res Clin Rheumatol. 2008; 22(4):759-72.

14. Wanyonyi N, Frantz J. Prevalence of work-related musculoskeletal disorders in Africa: a systematic review. Physiotherapy. 2015;101:e1604-5.

15. Temesgen MH, Belay GJ, Gelaw AY, Janakiraman B, Animut Y. Burden of shoulder and/neck pain among school teachers in Ethiopia. BMC Musculoskelet Disord. 2019;20(1):18

16. Kebede Deyyas W, Tafese A. Environmental and organizational factors associated with elbow/forearm and hand/wrist disorder among sewing machine operators of garment industry in Ethiopia. J Environ Public Health. 2014;2014.

17. Dheresa M. Geda B. Eastern Ethiopia: Low back pain and associated factors among nurses working in public hospitals of Harari region and Dire Dawa City administration; 2018.

18. Yosef T, Belachew A, Tefera Y. Magnitude and contributing factors of low back pain among long distance truck drivers at Modjo dry port, Ethiopia: a cross-sectional study. J Environ Public Health. 2019;2019.

19. Mekonnen TH. Work-related factors associated with low back pain among nurse professionals in east and west Wollega zones, Western Ethiopia, 2017: a cross-sectional study. Pain Therapy. 2019;8(2):239-47.

20. Wami SD, Dessie A, Chercos DH. The impact of work-related risk factors on the development of neck and upper limb pain among low wage hotel housekeepers in Gondar town, Northwest Ethiopia: institution-based crosssectional study. Environ Health Prev Med. 2019;24(1):27.

21. Tafese A, Nega A, Kifle M, Kebede W. Predictors of occupational exposure to neck and shoulder musculoskeletal disorders among sewing machine operators of garment industries in Ethiopia. Sci J Public Health. 2014;2(6): 577-83.

22. Abraha TH, Demoz AT, Moges HG, Ahmmed AN. Predictors of back disorder among Almeda textile factory workers, North Ethiopia. BMC Res Notes. 2018:11(1):304.

23. Moher D, Shamseer L, Clarke M, Ghersi D, Liberati A, Petticrew M, et al. Preferred reporting items for systematic review and meta-analysis protocols (PRISMA-P) 2015 statement. Syst Rev. 2015;4(1):1

24. Shamseer $L$, Moher $D$, Clarke M, Ghersi D, Liberati A, Petticrew $M$, et al. Preferred reporting items for systematic review and meta-analysis protocols (PRISMA-P) 2015: elaboration and explanation. BMJ. 2015:349:g7647.

25. Stroup DF, Berlin JA, Morton SC, Olkin I, Williamson GD, Rennie D, et al. Meta-analysis of observational studies in epidemiology: a proposal for reporting. Jama. 2000;283(15):2008-12.

26. Cohn J. Statistical power analysis for the behavioral sciences. Hillsdale, NJ: Lawrence Earlbam Associates; 1988

27. Luchini C, Stubbs B, Solmi M, Veronese N. Assessing the quality of studies in meta-analyses: advantages and limitations of the Newcastle Ottawa scale. World J Meta-Anal. 2017;5(4):80-4.

28. https://wellcomeopenresearch.s3.amazonaws.com/supplementary/13880/ ea30a2fba15a-44a9-b35e-5f0914db80b3.docx]. Accessed 1 Feb 2019. Google Search [Internet]. [cited 2020 Jan 11].

29. Doi SA, Thalib L. A quality-effects model for meta-analysis. Epidemiology. 2008:94-100.

30. Berkey CS, Hoaglin DC, Mosteller F, Colditz GA. A random-effects regression model for meta-analysis. Stat Med. 1995;14(4):395-411.

31. Higgins JP, Thompson SG. Quantifying heterogeneity in a meta-analysis. Stat Med. 2002;21(11):1539-58.

32. Stroup DF, Berlin JA, Morton SC, Olkin I, Williamson GD, Rennie D, et al. Meta-analysis of observational studies in epidemiology: a proposal for reporting. JAMA. 2000:283(15):2008-12.

33. Liu JL. The role of the funnel plot in detecting publication and related biases in meta-analysis. Evidence-based dentistry. 2011:12(4):121.

34. Sterne JA, Egger M. Regression methods to detect publication and other bias in meta-analysis. Publication bias in meta-analysis: prevention, assessment and adjustments. 2005:99-110.

35. Barendregt JJ, Doi SA, Lee YY, Norman RE, Vos T. Meta-analysis of prevalence. J Epidemiol Community Health. 2013;67(11):974-8.

36. Barendregt J, Doi S. MetaXL user guide version 5.3. EpiGear International Pty Ltd. 2016;

37. Nyaga VN, Arbyn M, Aerts M. Metaprop: a Stata command to perform metaanalysis of binomial data. Arch Public Health. 2014;72(1):39.

38. Louw QA, Morris LD, Grimmer-Somers K. The prevalence of low back pain in Africa: a systematic review. BMC Musculoskelet Disord. 2007:8(1):105.

\section{Publisher's Note}

Springer Nature remains neutral with regard to jurisdictional claims in published maps and institutional affiliations.

Ready to submit your research? Choose BMC and benefit from:

- fast, convenient online submission

- thorough peer review by experienced researchers in your field

- rapid publication on acceptance

- support for research data, including large and complex data types

- gold Open Access which fosters wider collaboration and increased citations

- maximum visibility for your research: over $100 \mathrm{M}$ website views per year

At BMC, research is always in progress.

Learn more biomedcentral.com/submission 